\title{
The deformation of a free surface due to the impact of a water droplet $^{\dagger}$
}

\author{
Sun-Hong Kwon ${ }^{1}$, Chang-Woo Park ${ }^{1}$, Seung-Hun Lee ${ }^{1}$, Jae-Young Shin ${ }^{1}$, \\ Young-Myung Choi ${ }^{1}$, Jang-Young Chung ${ }^{1}$ and Hiroshi Isshiki ${ }^{2 *}$ \\ ${ }^{1}$ Department of NAOE, Pusan National University, Busan, Republic of Korea \\ ${ }^{2}$ School of NAOE, University of Ulsan, Ulsan, Republic of Korea
}

(Manuscript Received November 28, 2010; Revised January 5, 2011; Accepted February 8, 2011)

\begin{abstract}
An attempt was made to compute the free surface deformation due to the impact of a water droplet. The Cauchy Poisson, i.e. the initial value problem, was solved with the kinematic and dynamic free surface boundary conditions linearized. The zero order Hankel transformation and Laplace transform were applied to the related equations. The initial condition for the free surface profile was derived from a captured video image. The effect of the surface tension was not significant with the water mass used in this investigation. The computed and observed free surface deformations were compared.
\end{abstract}

Keywords: Free surface deformation, bubble generation, Hankel transformation, Laplace transformation, Surface tension effect

\section{Introduction}

The authors observed bubbles generated on the free surface and in the water while they were performing sloshing experiment. Those generated bubbles influence a lot on the magnitude of the impact pressure. Fig. 1 shows the effect of bubbles on the impact pressure. In the left column the flow field captured by video camera is presented. In the upper picture there was no bubble on the free surface. However, the flow field in the lower contains a lot of bubbles in the water. The first peak shown in the figure was not affected by bubbles and air pocket. Those peaks following the first peak are quite different from the first peak. There are two distinct differences between them. The magnitude of the peaks with bubbles and air pocket are small-

\footnotetext{
tThis paper was modified from the abstract submitted for presentation to 26th International Workshop on Water Waves and Floating, 17-20 April 2011, Athens Greece.

* Corresponding author. Tel.: +82-51-510-2347, Fax: +82-51-581-3718.

E-mail address: shkwon@pusan.ac.kr.

Copyright (C) KSOE 2011.
}

er than that of the first peak. The pressure signals due to bubbles and peaks are oscillatory. Therefore investigation of bubble generation mechanism is very important in sloshing research. To investigate the bubble generation mechanism the authors started with very simple experiment. A water droplet was released at various heights above the calm water surface. Experiments were done for various masses to see mass effect on the free surface deformation. To investigate the effect of surface tension experiments with and without surfactant were done. During this investigation it was desired to compute the deformation of free surface due to a water droplet impact. This problem can be considered to be initial value problem, i.e., the classical Cauchy-Poisson problem. The deformation of free surface was recorded by high speed camera. This measured free surface deformation was used as initial condition for this initial value problem. The order zero Hankel transformation and Laplace integral transform was applied to the governing 
equation, kinematic and dynamic boundary conditions. Combining these results the expression for the free surface elevation can be obtained. The computed deformation of the free surface was compared with that of the experiment.

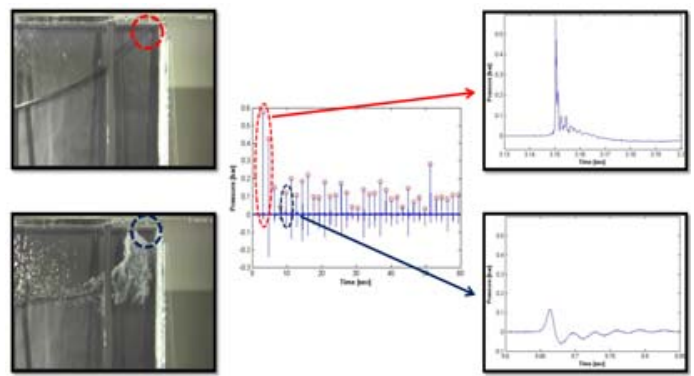

Fig.1 Flow fields and pressure time histories

\section{Experiment}

The experiment was carried out in a small wave tank whose dimensions are $60 \mathrm{~mm} \times 60 \mathrm{~mm} \times 60 \mathrm{~mm}$ $(\mathrm{L} \times \mathrm{B} \times \mathrm{H})$. The deformation of free surface was recorded by high speed camera. The maximum frame rate of the high speed camera is 64,000 rames/s. The frame rate for this study was 2,000 frames/s \& 1,000 frames/s. The experimental facility is shown in Fig. 2. Two different masses of water drops were tested to see the effect of the variation of the mass on the free surface deformation. To investigate the effect of the surface tension experiments with and without surfactant were done. To reduce the surface tension, NP-10 solution was added to the water. The surface tension was reduced from 72.7 dyne/cm to 36.5 dyne/cm after the solution was added. Fig. 3 shows the free surface profiles with and without surfactant when the mass of the water drop was $16 \mathrm{mg}$. The height of the drop origin was $150 \mathrm{~mm}$ above the calm water surface. Figs. 4 present the free surface profile when the mass of the water drop was $65 \mathrm{mg}$.

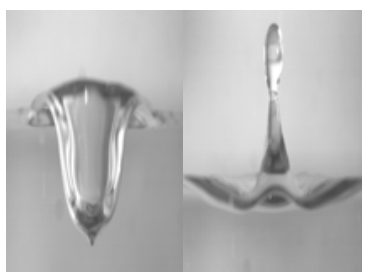

Without Surfactant



With Surfactant
Fig. 3 Comparison of the Effect of Surfactant (16mg)

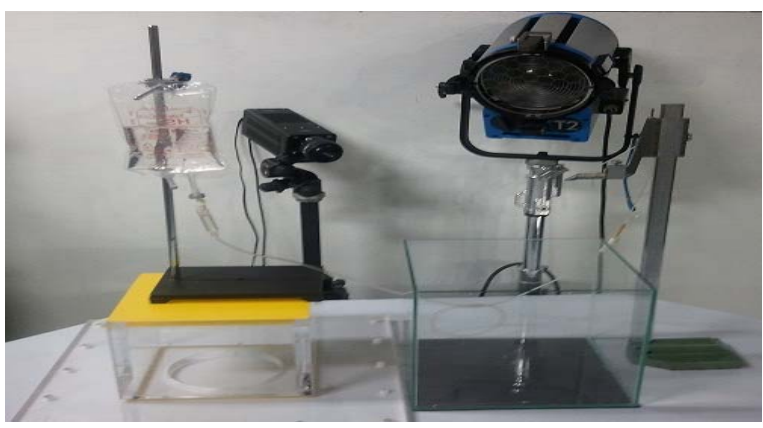

Fig.2 The Experimental Facility

The same drop height was maintained for both experiments. It can be shown that the difference due to mass effect gets smaller when the mass gets heavier. It means that when the size of disturbance gets larger the effect of surface tension gets smaller.

\section{Theoretical approach (Debnath, 1994)}

The fluid was assumed to be ideal. The irrotational flow motion was assumed. Based on these assumptions we can introduce velocity potential in the present analysis. The velocity potential is a function of $\mathrm{r}, \mathrm{z}$, and $\mathrm{t}$ under the assumption of axisymmetric motion of the free surface. The governing equation can be written in a form

$$
\frac{\partial^{2} \phi}{\partial r^{2}}+\frac{1}{r} \frac{\partial \phi}{\partial r}+\frac{\partial^{2} \phi}{\partial z^{2}}=0
$$

On the free surface two boundary conditions are needed to be imposed. The linearized kinematic free surface boundary condition is given.

$$
\frac{\partial \eta}{\partial t}=\frac{\partial \phi}{\partial z} \quad \text { on } \quad z=0
$$

The linearized dynamic free surface boundary condition is

$$
\frac{\partial \phi}{\partial t}+g \eta=0 \quad \text { on } \quad \mathrm{z}=0
$$

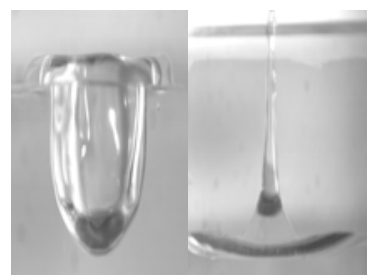

Without Surfactant

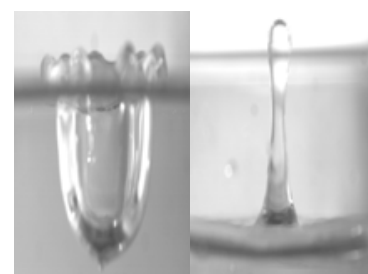

With Surfactant
Fig. 4 Comparison of the Effect of Surfactant (65mg) 
Along with these boundary conditions, we need to specify initial conditions for surface elevation and potential. To solve this problem integral transformation method is employed.

Application of order zero Hankel and Laplace transform to velocity potential is defined by

$$
\bar{\phi}(k, z, s)=\int_{0}^{\infty} e^{-s t} d t \int_{0}^{\infty} r J_{0}(k r) \phi(r, z, t) d r
$$

Its inverse can be deified by

$$
\phi(r, z, t)=\frac{1}{2 \pi i} \int_{\Gamma} e^{s t} d s \int_{0}^{\infty} k J_{0}(k r) \bar{\phi}(k, z, s) d k
$$

Application of Eq. (4) to Eq. (1) yields (Dautray, 1985)

$$
-k^{2} \bar{\phi}(k, z, s)+\frac{d^{2} \bar{\phi}(k, z, s)}{d z^{2}}=0
$$

When we consider $\mathrm{z}<0$, we can have the solution under deep water assumption as

$$
\bar{\phi}(k, z, s)=A(k, s) e^{k z}
$$

Transformation of Eq. (2) gives us

$$
s \bar{\eta}(k, s)-\bar{\eta}(k, 0)=k A(k, s) \quad \text { on } z=0
$$

Eq. (3) becomes

$$
s A(k, s)-\bar{\phi}(k, 0,0)+g \bar{\eta}(k, s)=0 \text { on } z=0
$$

We can write the expression for the elevation by solving Eq. (8) and Eq. (9) with an assumption of banishing of initial potential $\bar{\phi}(k, 0,0)$.

$$
\bar{\eta}(k, s)=\frac{1}{s^{2}+g k} s \bar{\eta}(k, 0) \quad \text { on } \quad z=0
$$

Inversion of Hankel Laplace transformation will give us the free surface elevation

$$
\eta(r, t)=\int_{0}^{\infty} k J_{0}(k r) \bar{\eta}(k, 0) \cos \sqrt{g k} t d k
$$

Let's consider the initial displacement

$$
\eta(r, 0)=d e^{-\left(\frac{r}{a}\right)^{2}}\left\{1-\left(\frac{r}{a}\right)^{2}\right\}
$$

The Hankel transform of Eq. (12) results in (Debnath, 1994 ; Miles, 1968)

$$
\bar{\eta}(k, 0)=\frac{1}{8} d a^{2}(k a)^{2} e^{-\frac{1}{4}(k a)^{2}}
$$

Substitution of Eq. (13) into (11) gives solution for the free surface elevation in a form

$$
\eta(r, t)=\frac{1}{8} d a^{4} \int_{0}^{\infty} k^{3} J_{0}(k r) e^{-\frac{1}{4}(k a)^{2}} \cos \sqrt{g k} t d k
$$

\section{Result and discussion}

The related parameters of Eq. (12) are 'a' and ' $d$ '. These parameters were computed from the digitized free surface deformation captured by high speed camera. The two curves are shown in Fig. 5. The symbols represent measured free surface deformation. The solid line shows the curves calculated using Eq. (12). The units of the axes are $\mathrm{cm}$. Having known these ' $a$ ' and ' $d$ ' values we can compute the free surface deformation making use of Eq. (14). The computed elevation moves much slower than that of measured one. The maximum surface elevations for both computed and measured are shown in Fig. 6. It seems that the slope of the computed results is smaller than that of the measurement. For the $16 \mathrm{mg}$ case the deviation with surfactant is smaller. It seems that this is due to the fact that the computation did not include the surface tension effect in the dynamic free surface boundary condition. The computed profile of the free surface deviates a lot at the end of the surface elevation. The reason of the deviation might be due to some factors. First of all, the calculation was based on the potential theory which ignores the effect of viscosity.


Fig.5 Initial Condition for the Free Surface Deformation 

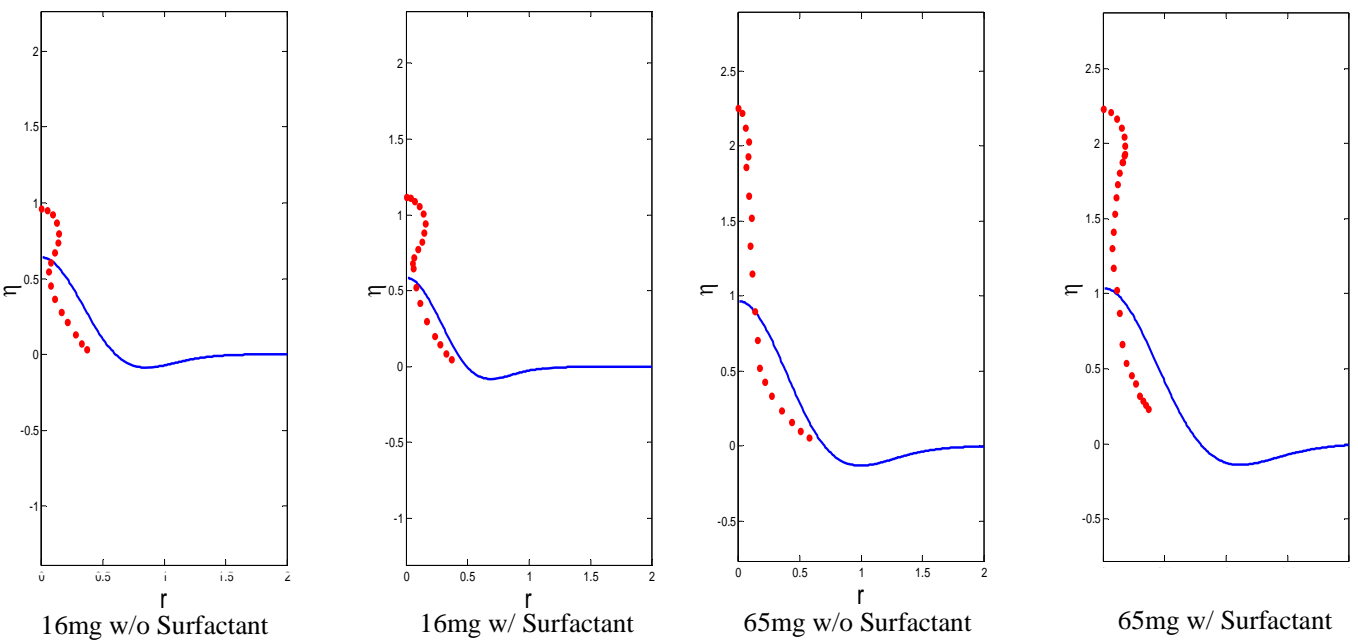

Fig. 6 Comparison of the Maximum Surface Elevations.

Secondly the free surface boundary conditions are linearized. Thirdly the surface tension was not included in the dynamic free surface boundary condition.

In addition to the above mentioned reasons, the initial free surface deformation calculated from Eq. (12) does not match well with the measured profile which is featured in Fig. 5.

\section{Conclusions}

The free surface deformation due to water droplet impact was calculated. The Cauchy-Poisson initial value problem was solved by making use of the video captured images of the free surface. The order zero Hankel transform and Laplace transform were applied to the governing equation and free surface boundary conditions. The derived free surface profile cannot be obtained in a closed form solution. Numerical solution was presented. It turned out that the effect of the surface tension was not significant with the magnitude of the water mass used in this investigation. The computed and observed free surface deformations were compared. The computed and measured free surface profiles were not in good agreement. The reasons of this discrepancy were presented in the results and discussion section of the present paper.

\section{Acknowledgements}

This work was supported by the National Research Foundation of Korea(NRF) grant funded by the Korea government.(NRF-2010-0025457)

\section{References}

[1] Robert Dautray, Jacques-Louis Lions, Mathematical analysis and numerical methods for science and technology,vol.2, functional analysis and variational methods, Springer(1985)

[2] Lokenath Debnath, Nonlinear water waves, Academic Press, INC(1994)

[3] John W.Miles, The Cauchy-Poisson problem for a viscous liquid, J. Fluid Mech. Vol.34, part2, (1968) 359-370 